\title{
Particle Swarm Optimization-based BP Neural Network for UHV DC Insulator Pollution Forecasting
}

\author{
Fangcheng Lü ${ }^{1}$, Chunxu Qin ${ }^{1, *}$, Yunpeng $^{1}$ \\ ${ }^{1}$ Hebei Provincial Key Laboratory of Power Transmission Equipment Security Defense, North China Electric Power University, \\ Baoding071000, China
}

Received 15 June 2013; Accepted 23 February 2014

\begin{abstract}
In order to realize the forecasting of the UHV DC insulator's pollution conditions, we introduced a PSOBP algorithm. A BP neural network (BPNN) with leakage current, temperature, relative humidity and dew point as input neurons, and ESDD as output neuron was built to forecast the ESDD. The PSO was used to optimize the the BPNN, which had great improved the convergence rate of the BP neural network. The dew point as a brand new input unit has improved the iteration speed of the PSOBP algorithm in this study. It was the first time that the PSOBP algorithm was applied to the UHV DC insulator pollution forecasting. The experiment results showed that the method had great advantages in accuracy and speed of convergence. The research showed that this algorithm was suitable for the UHV DC insulator pollution forecasting.
\end{abstract}

Keywords: PSOBP, UHVDC, Insulator Pollution, Forecasting

\section{Introduction}

The $\pm 800 \mathrm{kV}$ UHV DC power transmission projects are being developed quickly in China. The UHV DC transmission project provides a range of advantages, not only does this benefit the power transmission with large-capacity and longdistance and spare many transmission corridors, but it also improves the security of the Power Grid [1-4]. The insulator is essential equipment in power system. The flashover of the insulator is a major problem to the power grid safety. The measurement and cleanout of the insulator pollution have great importance to protect the power grid from the flashover. The insulator pollution measurement results usually can be expressed as Equivalent Salt Deposit Density (ESDD) and Non-soluble Deposit Density (NSDD), and the ESDD is very important to the insulator flashover [5]. Researches on outdoor insulation showed the pollution of $\mathrm{DC}$ insulators is much more serious than that of $\mathrm{AC}$ insulators $[6,7]$. So the on-line monitoring of the UHV DC insulator's ESDD is more important.

Now the on-line monitoring of insulator leakage currents has been realized, and the meteorological parameters (such as the temperature, humidity and so on) can be on-line measured by the weather station, however, the insulator pollution measurement is done by manual work[8,9]. Studies $[10,11]$ showed that the leakage current of insulator was strongly linked to the ESDD and the operation meteorological environment of the insulator. Therefore, the ESDD can be forecasted by the leakage current and meteorological parameters. However, the relationship among the leakage current, ESDD, and meteorological parameters is

\footnotetext{
*E-mail address: fangchenglswcd@126.com

ISSN: 1791-2377 @ 2013 Kavala Institute of Technology. All rights reserved.
}

complex, so the prediction of ESDD by the leakage current and meteorological parameters is a complex nonlinear problem. To solve this problem, some researchers predicted the ESDD using the back propagation (BP) neural network [10-12].

The standard BP algorithm trains the weights and biases of neural network by the methods of gradient descent or conjugate gradient decent by calculating the partial derivative of the performance with respect to the weights and biases values. BPNN has been widely used in many fields. However, it has been proven that conventional BP algorithm has defects of slow convergent speed and easy convergence to a local minimum point of error function [13].

Particle swarm optimization (PSO) algorithm [14] is a random optimization method based on swarm intelligence which has more powerful ability of global optimization. It can be easily implemented, and does not require the gradient information of an objective function, but only the values. Therefore, the PSO algorithm is applied to the BP neural network in the training process, to obtain a set of weights that will minimize the error function in competitive time. The weights are progressively updated until the convergence criterion is satisfied.

In this study, firstly some basic concept of BPNN and PSO are briefly described. Then a algorithm that BPNN optimized by PSO is presented, which called PSOBP in our study. And then, the PSOBP is used to forecast the ESDD as a new application in the UHV DC projects.

\section{BP Neural Networks}

A sample neural network includes three different layers: the input layer, the hidden layer and the output layer. BPNN is a multi-layer feed forward neuron network, and the BP algorithm includes two components: the forward 
propagation of information and the back propagation of error. The input layer is responsible for receiving information from the outside, and the input information which has been handled by the hidden layer unit is transmitted to the output layer; while the output layer is responsible for transmitting the result of information processing to the outside. If the result in the output layer is not ideal, the network will calculate the variation of errors and propagate errors back along the former route while correcting the weight. This process is repeated until it gets the satisfied result or it reaches the maximum number of iterations. Therefore, BP algorithm is also called as the back propagation of error. Although BP network has been widely used, it has serious flaws of slow convergence, prone to local minima, poor generalization and etc. The sample topology of neural network is shown in Fig. 1.

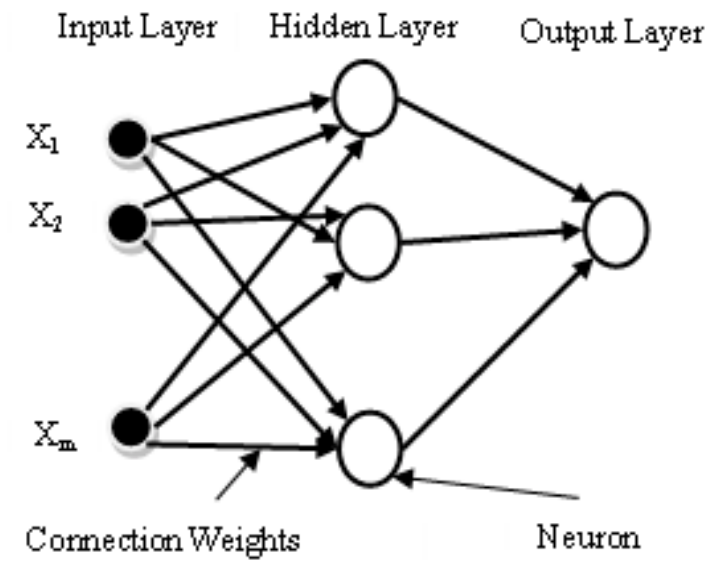

Fig.1. A simple BP neural network

\section{Particle Swarm Optimization}

Particle swarm optimization is firstly introduced by Kennedy and Eberhart in the year of 1995 [14] as an evolutionary computation method used to deal with the optimization problems. The PSO algorithm is inspired from the sociological and biological behavior of flocks of birds searching for their food. Because of clear concepts, fast arithmetic speed, and easy to be realize, PSO has been used to a lot of research areas and engineering fields, such as optimization of complex function, training of artificial neural network, and control of fuzzy system.

In the beginning the system has a lot of random solutions. Each particle is a potential solution and randomly given an initial velocity and pass through the searching space. Each particle finds the global best position by competition and cooperation among the particles in iterative process. Supposing D is the dimension of searching space, $\mathrm{N}$ is the total number of particles, the particle position of the $i^{\text {th }}$ iteration is expressed as $X_{i}=\left(x_{i 1}, x_{i 2}, \ldots, x_{i D}\right)$; the particle velocity of the $i^{\text {th }}$ is represented as $V_{i}=\left(v_{i 1}, v_{i 2}, \ldots, v_{i D}\right)$; the best position of the ith particle is denoted as $P_{i}=\left(p_{i 1}, p_{i 2}, \ldots, p_{i D}\right) ;$ and the best position of the whole swarm is denoted as $P_{g}=\left(p_{g 1}, p_{g 2}, \ldots, p_{g D}\right)$
In order to balance the global search and local search of PSO algorithm, a inertia weight was introduced by Shi and Eberhart $[15,16]$. Therefore the particle updates its velocity and positions according to the following equations:

$$
\begin{aligned}
& v_{i d}^{k+1}=w(k) v_{i d}^{k}+c_{1} r_{1}\left(p_{i d}^{k}-x_{i d}^{k}\right)+c_{2} r_{2}\left(p_{g d}^{k}-x_{i d}^{k}\right) \\
& x_{i d}^{k+1}=x_{i d}^{k}+v_{i d}^{k+1} \\
& \mathrm{i}=1,2, \ldots, \mathrm{N} \\
& \mathrm{k}=1,2, \ldots, \mathrm{T}_{\max }
\end{aligned}
$$

Where

$\mathrm{N}$ is the number of particles in population,

$\mathrm{k}$ is the current iterative number,

$\mathrm{T}_{\max }$ is the number of iterative generation,

$\mathrm{c} 1$ and $\mathrm{c} 2$ are the cognitive and social acceleration constants, $\mathrm{r} 1$ and $\mathrm{r} 2$ are uniformly distributed random numbers in the range $[0,1]$,

$v_{i d}^{k}$ and $v_{i d}^{k}$ are current and modified velocity for each iteration, respectively,

$x_{i d}^{k}$ and $x_{i d}^{k+1}$ are the current and modified position for each iteration respectively,

$w(k)$ is called the inertia factor described by following equation,

$w(k)=w_{\text {max }}-\left(w_{\max }-w_{\min }\right) \frac{k}{T_{\max }}$

where $w(k)$ decreases linearly from $w_{\max }$ to $w_{\min }$ in a iterative process.

And the particle velocity is limited to in [Vmin, Vmax].

\section{PSOBP Algorithm}

The neural network performance function is normally chosen to be the mean squared error (MSE) for each pattern on the training set

$$
M S E=\frac{1}{N} \sum_{i=1}^{i=N} \sum_{j=1}^{j=C}\left(y_{i j}^{d}-y_{i j}\right)^{2}
$$

Where,

$$
\begin{aligned}
& y_{i j}^{d} \text { is real output of the network, } \\
& y_{i j} \text { is the predicted output of the network }
\end{aligned}
$$

$\mathrm{N}$ is the total number of training samples,

$\mathrm{C}$ is the number of output neurons.

The particle fitness value can be calculated by (4).

The PSOBP NN is described as follows. Each dimension of the particle corresponds to a neural network connection weight or threshold. So the dimension of the particle can be express as follows:

$$
D=d^{*} m+m * n+m+n
$$

where 
$\mathrm{d}$ is the number of input neurons,

$\mathrm{m}$ is the number of hidden layer's neurons,

$\mathrm{n}$ is the number of output neurons.

The procedure of the PSOBP can be described as follows:

Step1: Initializing topology of $\mathrm{BP} \mathrm{NN}$ according to the training sample set.

Step2: Setting the dimension and population size of the particles, selectting fittness function, the inertia factor and the maximum iteration times. Randomly initializing the velocity and position of each particle.

Step3: Calculating fitness value of each particle by (4). The $\mathrm{P}_{\mathrm{b}}$ is selected as the positions of the current particles and $\mathrm{P}_{\mathrm{g}}$ is selected as the best position of all the particles. If current fitness value is less than the $\mathrm{P}_{\mathrm{b}}$ fitness value, the current one replaces the $P_{b}$.

Step4: The best particle of the current particles is stored. The velocity and position of each particle is updated by (1) and (2), and then generate the new particles. If velocity or position of particle is beyond the boundary, the velocity or position should be resetted randomly.

Step5: Evaluate each new particle's fitness value. If the ith particle's new position is better than $\mathrm{P}_{\mathrm{ib}}, \mathrm{P}_{\mathrm{ib}}$ is selected as the new position of the ith particle. If the best position of all new particles is better than $P_{g}$, then $P_{g}$ is updated.

Step6: If the maximal iteration times or the fitness values are met, stop the iteration, and the positions of particles represented by $\mathrm{P}_{\mathrm{g}}$ are the optimal best solution. Otherwise, the process is repeated from step3.

Step7: Taking the weights and threshold values which optimized by PSO as the initial parameters, the BP network makes autonomous learning.

\section{Applications in Forecasting of UHV DC Insulator's ESDD}

\subsection{Samples selected}

Previous studies [10-12] showed that the leakage current of insulator has close association with the temperature, relative humidity and the ESDD. And they selected the temperature, relative humidity and leakage current as the input units of BP neural network. However, in this study, we selected the leakage current, the temperature, relative humidity and the dew point as the input units, and the ESDD of one porcelain UHV DC insulator was selected as the output units. The dew point was selected as a new input unit in our study.

The on-line monitoring technology of the leakage current, the temperature, relative humidity and the dew point can refer to [8], and the measurement of the ESDD and its results can refer to [9]. The data of the leakage currents, the temperature, relative humidity and the dew point are synchronous. The data set includes 671 data points, of which 480 points were used to train the BPNN and the rest will be used to test. The training data were collected on the day just before the ESDD measurement, and the test data were collection around the day on which the ESDD measured but not the day just before the ESDD measurement. Because the insulator pollution accumulated very slowly, the ESDD value changed very little in these days. All the data are from the UHV DC insulator test system and the details can refer to $[8]$.

\subsection{The network topology of neuron networks}

In this study, the BP neuron network has three layers including one hidden-layer. The neural networks models are trained with 4 neurons as input data while 3 neurons for the hidden layer, and 1 neuron for output layer. The neuron transferring function in hidden-layer is tansig, and that in output-layer is purelin. And the training function is traingdm. The training error precision is 0.0001 .

\subsection{Parameters of PSO}

The parameters of PSO were selected as follows. The initial location and velocity of search point is randomly generated between $[-1,1]$; the maximum velocity of particles is 0.5 ; the population size is 40 ; the maximum times of iteration is 30000 ; the accelerated coefficients $\mathrm{c} 1=2.3, \mathrm{c} 2=1.8$; the inertia weight is gradually decreased from 0.90 to 0.40 by (3) in order to reduce the influence of past velocity, and according to (5) the particle dimension is 19 .

\subsection{Training Performance}

The performances of three forecasting models, including the traditional BP neural network, the PSOBP algorithm with the leakage current, the temperature, relative humidity and the dew point as the input units, and the PSOBP algorithm with no the dew point.

The convergence rate of three forecasting models are shown in Fig.2, Fig.3 and Fig.4, respectively. Form these figures, the best training performance of the traditional BP neural network is $9.9997 \times 10^{-5}$ at epoch 23779 , that of the PSOBP algorithm is $9.8399 \times 10^{-5}$ at epoch 119 , and that of the PSOBP algorithm with no the dew point is $9.6847 \times 10^{-5}$ at epoch 1212. The iterations of PSOBP algorithm is only less than $1 \%$ that of the traditional BP neural network's. So it could be seen that the number of PSOBP algorithm's iterations is much less than that of the BP neural network's, which means that the convergence rate of the PSOBP algorithm is much faster than that of the traditional BP network. The introduce of the dew point has great improved the arithmetic speed from the Fig.3 and Fig.4.

From the above, the PSOBP algorithm with the leakage current, the temperature, relative humidity and the dew point as the input units, has a great advantage in the arithmetic speed over the other two models.

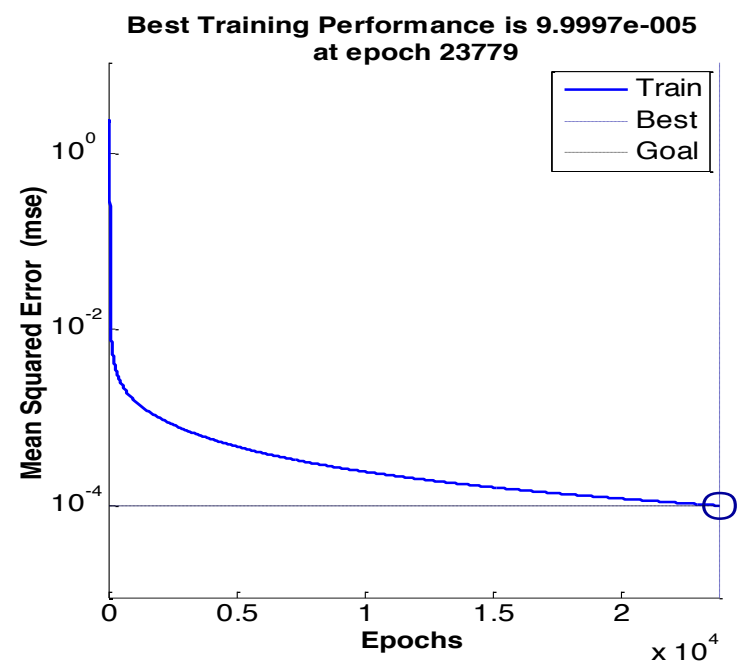

Fig.2. Convergence rate of the BP neural network 


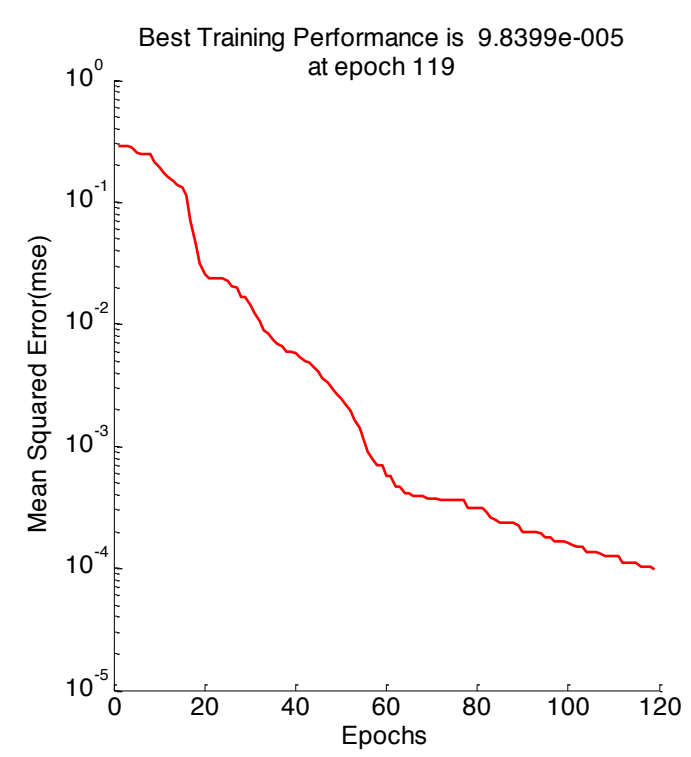

Fig.3. Convergence rate of the PSOBP algorithm

The training accuracy of the PSOBP algorithm is shown in the Fig.5, and the training relative errors of this method are shown in Fig.6. From these figures, training values of the ESDD are close to the real values, and the training relative errors are less than $0.5 \%$. It can be seen that the training performance of the PSOBP algorithm is perfect in our study.

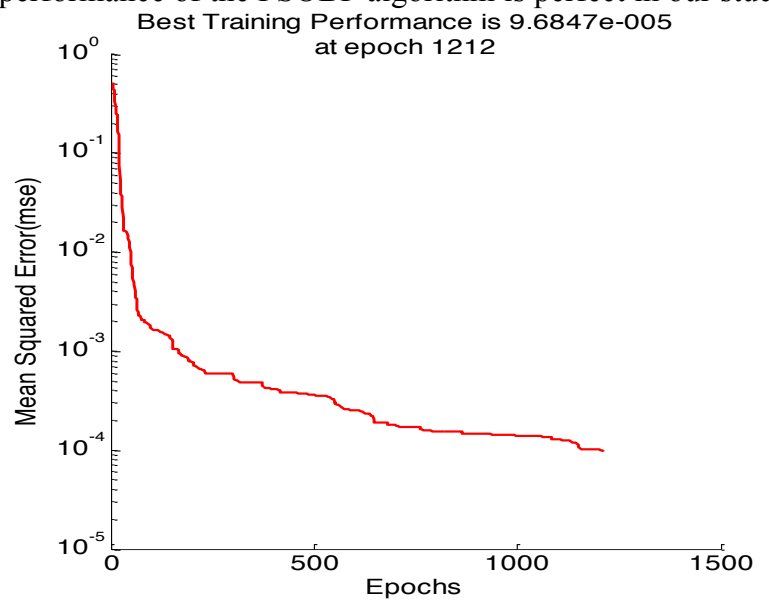

Fig.4. Convergence rate of the PSOBP algorithm with no the dew point

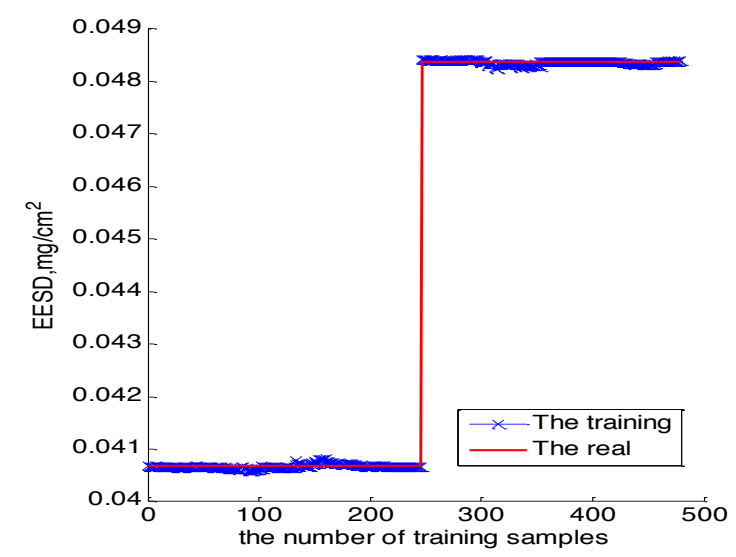

Fig.5.The training performance of the PSOBP algorithm

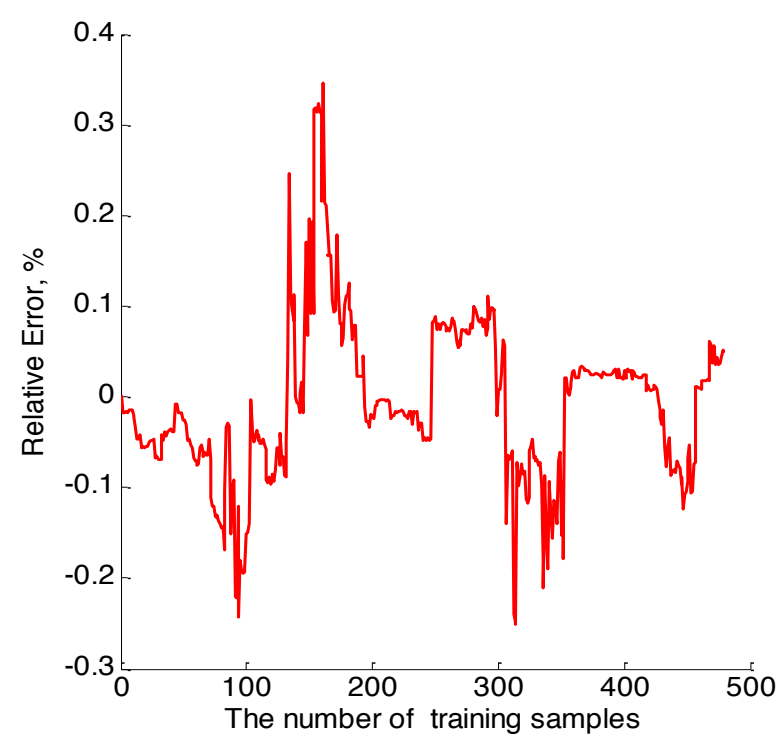

Fig.6.The training relative errors of the PSOBP algorithm

\subsection{Forecasting with the PSOBP}

The forecasting values of the ESDD with PSOBP algorithm are shown in Fig.7, and the forecasting relative errors of this method are shown in Fig.8. In these figures, the forecasting values are close to the real values, and the relative errors are less than $0.5 \%$. The forecasting results show perfect, and it shows that the PSOBP algorithm is a good method for the forecasting ESDD of UHV DC insulator.

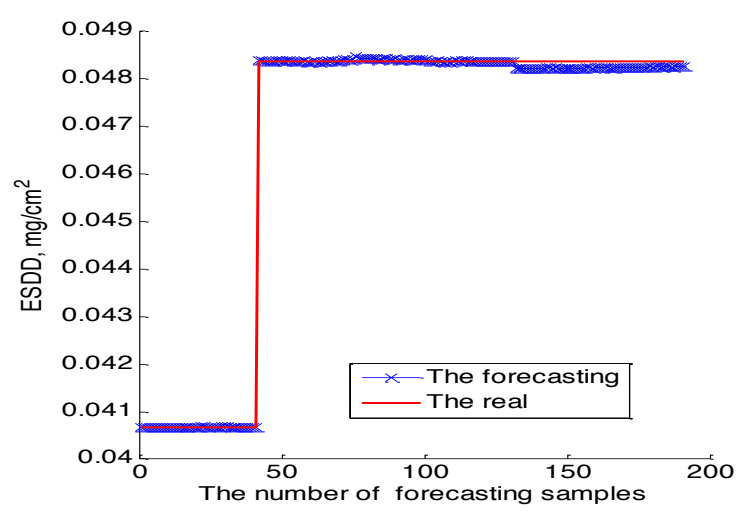

Fig.7. The forecasting results of the ESDD with the PSOBP algorithm

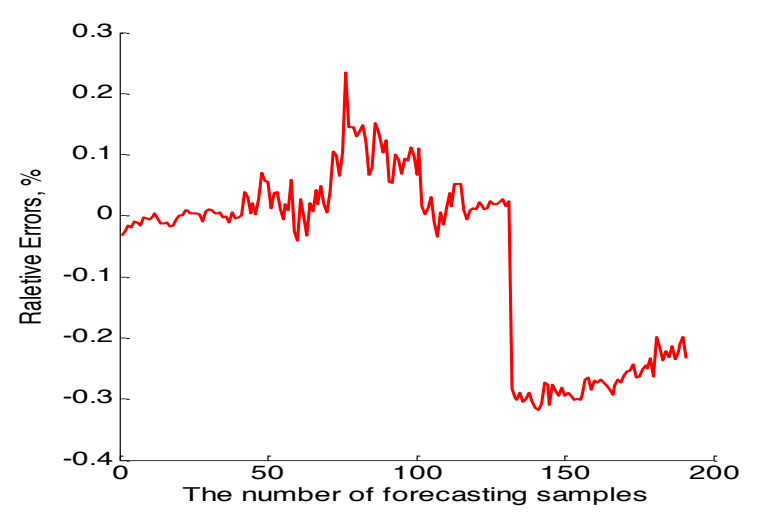

Fig.8. The relative errors of the ESDD forecasting with the PSOBP algorithm 


\section{Conclusions}

In this paper, we have proposed a new application of the PSOBP algorithm that forecasts the ESDD of the UHV DC insulators. The convergence rate of the BP neural network had been greatly improved by the PSO algorithm. The iteration speed of the PSOBP algorithm can be improved significantly with the introduce of the dew point. The forecasting results showed perfect, the relative errors of the forecasting are less than $0.5 \%$. The main benefit of the forecasting model based on the PSOBP algorithm is fast speed and accuracy. Therefore, the ESDD forecasting of
UHV DC insulator based on the PSOBP algorithm will be used in the practical engineering in future.

\section{Acknowledgments}

This work was supported by the National Science Found of China (51207055), the Fundamental Research Funds for the Central Universities (13ZD14) and scientific and technological projects of China Southern Power Grid (k201016).

\section{References}

1. LI Li-cheng, "Technical characteristics and engineering applications of UHVDC power transmission", China Southern Power Grid Technology Research, 2005,pp.1-13.

2. SHU Yin-biao, LIU Ze-hong, GAO Li-ying, et al, "A preliminary exploration for design of $\pm 800 \mathrm{kV}$ UHVDC project with transmission capacity of $6400 \mathrm{MW}$," Power System Technology, 2006, pp.1-8.

3. SHU Yin-biao, ZHANG Wen-liang, "Research of key technologies for UHV transmission," Proceedings of the CSEE, 2007, pp.1-6.

4. Yin-biao SHU, "Development and execution of UHV power transmission in China," Electric Power, 38(11), 2005, pp.1-8.

5. WANG Tao, OU Qi-he, WU Jiang-hong, LI Jing-dong, YAO Sheng, "TEST AND RESEARCH ON CLEANING OF HIGH VOLTAGE TRANSMISSION LINE BASED ON SALT DENSITY," Power System Technology, 28(4), 2004, pp.22-26.

6. Z.Y. Su, Y. S. Liu. "Comparison of natural contaminants accumulated on surfaces of suspension and post insulator with DC and AC stress in northern China's inland areas," Power System Technology, 10, 2004, pp.13-17.

7. T. C. Cheng, C. T. Wu, J. N. Rippey, et al. "Pollution Performance of DC Insulators under Operating Conditions," IEEE Transactions on Electrical Insulation, 6, 1981, pp.154-164.

8. Fangcheng Lü, Chunxu Qin, Yunpeng Liu and Wenyi Guo, "Study on the Ultra-high Voltage DC Insulators Long-term Running Research Platform," Advanced Materials Research , 2013, pp.17821785
9. Fangcheng Lv, Chunxu Qin, Yunpeng Liu, Wenyi Guo, Ruihai Li, "A Comparative Study on Contamination Deposited Characteristics of $\pm 800 \mathrm{kV}$ DC Line Insulators," International Journal of Computer Science Issues, Vol. 10, Issue 1, No 3, January 2013, pp.37-43.

10. MAO Ying-ke, GUAN Zhi-cheng, WANG Li-ming, YUE Bo , "Prediction of Leakage Current of Outdoor Insulators Based on BP Artificial Neural Network," Proceedings of the CSEE, Vol.27, No.27, Sep. 2007, pp.7-12.

11. Zhang han, Wen xishan, Ding hui, "Extrapolation of Insulator's ESDD based on Climate Factor with Artificial Neural Network ", High Voltage Apparatus, 39(6), 2003, pp.31-32,35.

12. HE Xiang-you, XIANG Feng-hong, HU Jian-rui, "Prediction of ESDD of Outdoor Insulators Based on BP Artificial Network with Fuzzy Output," Insulation Materials, 41(4), 2008, pp: 58-61.

13. D. E. Rumelhart, G. E. Hinton, and R. J. Williams, "Learning representation by back propagating errors," Nature, 1986, pp. 533536.

14. Kennedy J, Eberhart R C. "Particle swarm optimization," Proceedings of IEEE International Conference on Neural Networks. Piscataway, NJ, USA: IEEE Service Center, 1995, pp.1942-1948.

15. Shi Y, Eberhart R C. "A modified particle swarm optimizer," Proceedings of the IEEE International Conference on Evolutionary Computation, 1998, pp.69-73

16. Shi Y; Eberhart R C. "Fuzzy adaptive particle swarm optimization," Proceedings of the IEEE International Conference on Evolutionary Computation, 2001, pp.101-106. 